\title{
On Tuza's conjecture for graphs with treewidth at most 6
}

\author{
Fábio Botler ${ }^{1}$, Cristina G. Fernandes ${ }^{2}$, Juan Gutiérrez ${ }^{2}$ \\ ${ }^{1}$ CIMFAV, Facultad de Ingeniería \\ Universidad de Valparaíso, Valparaíso - Chile \\ fbotleredii.uchile.cl \\ ${ }^{2}$ Departamento de Ciência da Computação \\ Universidade de São Paulo - São Paulo, SP - Brazil \\ \{cris, juanguti\}aime.usp.br
}

\begin{abstract}
Tuza (1981) conjectured that the size $\tau(G)$ of a minimum set of edges that meets every triangle of a graph $G$ is at most twice the size $\nu(G)$ of a maximum set of edge-disjoint triangles of $G$. In this paper we verify this conjecture for graphs with treewidth at most 6 .
\end{abstract}

\section{Introduction}

In this paper, all graphs considered are simple and the notation and terminology are standard. A triangle transversal of a graph $G$ is a set of edges of $G$ whose deletion results in a triangle-free graph; and a triangle packing of $G$ is a set of edge-disjoint triangles of $G$. We denote by $\tau(G)$ (resp. $\nu(G)$ ) the size of a minimum triangle transversal (resp. triangle packing) of $G$. In [Tuza 1981] the following conjecture was posed:

Conjecture (Tuza, 1981). For every graph $G$, we have $\tau(G) \leq 2 \nu(G)$.

This conjecture was verified for many classes of graphs, in particular for planar graphs in [Tuza 1990], and tripartite graphs in [Haxell and Kohayakawa 1998]. A set of tools for dealing with graphs that contain vertices of small degree was introduced in [Puleo 2015] (Lemma 4), and Tuza's Conjecture was verified for graphs with maximum average degree less than 7 , i.e., for graphs in which every subgraph has average degree less than 7 . In this paper, we extend this technique (Lemma 5) in order to prove Tuza's Conjecture for graphs with treewidth at most 6 (Theorem 6). The following example (Figure 1) shows that there are graphs with treewidth at most 6 whose maximum average degree is at least 7. So our result is not implied by the previous ones. (Conversely, note that the $k \times k$ grid has treewidth at least $k$ and maximum average degree at most 4.) Due to space limitations, we present only a sketch of some proofs.

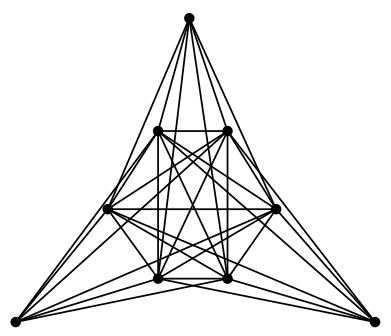

Figure 1. A graph with treewidth 6 and average degree 22/3. 
A rooted tree is a pair $(T, r)$, where $T$ is a tree and $r$ is a vertex of $T$. Given $t \in V(T)$, let $P_{T}(t)$ be the unique path in $T$ that joins $r$ and $t$. If $t^{\prime}$ is a vertex in $V\left(P_{T}(t)\right)$, then $t^{\prime}$ is an ancestor of $t$. Every vertex in $T$ that has $t$ as its ancestor is called a descendant of $t$. If $t \neq r$, then the parent of $t$, denoted by $p(t)$, is the ancestor of $t$ that is adjacent to $t$. The successors of $t$ are the vertices whose parent is $t$, and we denote the set of sucessors of $t$ by $S_{T}(t)$. The height of $t$, denoted by $h_{T}(t)$, is the length of a longest path that joins $t$ to a descendant of $t$. When $T$ is clear from the context, we simply write $S(t)$ and $h(t)$. For a graph $G$ and a vertex $v$ of $G$, we denote by $N_{G}(v)$ the set of neighbors of $v$ in $G$. When $G$ is clear from the context, we simply write $N(v)$.

A tree decomposition of a graph $G$ is a pair $(T, \mathcal{V})$, consisting of a tree $T$ and a collection $\mathcal{V}=\left\{V_{t}: t \in V(T)\right\}$ of bags $V_{t} \subseteq V(G)$, satisfying the following conditions:

(T1) $\bigcup_{t \in V(T)} V_{t}=V(G)$;

(T2) for every $u v \in E(G)$, there exists a bag $V_{t}$ such that $u, v \in V_{t}$;

(T3) if a vertex $v$ is in two different bags $V_{t_{1}}, V_{t_{2}}$, then $v \in V_{t}$ for every $t$ in the path of $T$ that joins $t_{1}$ and $t_{2}$.

The width of $(T, \mathcal{V})$ is the number $\max \left\{\left|V_{t}\right|-1: t \in V(T)\right\}$, and the treewidth $t w(G)$ of $G$ is the minimum width of any tree decomposition of $G$. We refer to the vertices of $T$ as nodes. If $G$ is a graph with treewidth $k$, then we say that $(T, \mathcal{V})$ is a full tree decomposition of $G$ if $\left|V_{t}\right|=k+1$ for every $t \in V(T)$, and $\left|V_{t} \cap V_{t^{\prime}}\right|=k$ for every $t t^{\prime} \in E(T)$. Every graph has a full tree decomposition (see [Bodlaender 1998, Gross 2014]).

We say that a triple $(\mathcal{V}, T, r)$ is a rooted tree decomposition of a graph $G$ if $(\mathcal{V}, T)$ is a full tree decomposition of $G,(T, r)$ is a rooted tree, and $V_{t} \cap V_{p(t)} \neq V_{t} \cap V_{t^{\prime}}$ for every $t \in V(T) \backslash\{r\}$ and $t^{\prime} \in S(t)$. Given a rooted tree decomposition $(\mathcal{V}, T, r)$ of a graph $G$, and a node $t \in V(T)$ with $t \neq r$, we say that the (unique) vertex $v_{t} \in V_{t} \backslash V_{p(t)}$ is the representative of $t$.

Proposition 1. Every graph has a rooted tree decomposition.

Proposition 2. If $t$ is a leaf of a rooted tree decomposition of a graph $G$ and $y$ is the representative of t, then $N_{G}(y) \subseteq V_{t}$.

\section{Graphs with treewidth at most 6}

A nonempty set $V_{0} \subseteq V(G)$ is called reducible if there is a set $X \subseteq E(G)$ and a set $Y$ of edge-disjoint triangles in $G$ such that the following conditions hold: $(i)|X| \leq 2|Y|$; (ii) every triangle containing a vertex of $V_{0}$ has an edge in $X$; and (iii) if $u v \in E(A)$ for some $A \in Y$, and $u, v \notin V_{0}$, then $u v \in X$. When $V_{0}, X$, and $Y$ satisfy the definition above, we say that $V_{0}$ is reducible using $X$ and $Y$. When $G$ has no reducible set, we say that $G$ is irreducible. The following lemma comes naturally.

Lemma 3 ([Puleo 2015, Lemma 2.2]). Let $G$ be a graph and $V_{0} \subseteq V(G)$ be reducible using $X$ and $Y$. Let $G^{\prime}=(G-X)-V_{0}$. If $\tau\left(G^{\prime}\right) \leq 2 \nu\left(G^{\prime}\right)$, then $\tau(G) \leq 2 \nu(G)$.

We say that a graph $G$ is robust if, for every $v \in V(G)$, every component of $G[N(v)]$ has order at least 5. The following lemma (see [Puleo 2015, Lemma 2.7]) is an important tool in our proof. In what follows, the closed neighborhood $N(u) \cup\{u\}$ of a vertex $u \in V(G)$ is denoted by $N[u]$ and $\Delta(G)$ is the maximum degree in $G$.

Lemma 4. If $G$ is an irreducible robust graph and $x, y \in V(G)$, then the following holds. 
(a) if $d(x) \leq 6$, then $\Delta(\overline{G[N(x)]}) \leq 1$ and $|E(\overline{G[N(x)]})| \neq 2$;

(b) if $d(x) \leq 6$ and $d(y) \leq 6$ then $x y \notin E(G)$;

(c) if $d(x)=7$ and $d(y)=6$ then $N[y] \nsubseteq N[x]$;

(d) if $d(x) \leq 8$ and $d(y)=5$, then $N[y] \nsubseteq N[x]$.

In this paper we extend the result above to the following lemma.

Lemma 5. If $G$ is an irreducible robust graph and $x, y \in V(G)$ are such that $x y \notin E(G)$, $d(x), d(y) \leq 6$, and $|N(x) \cup N(y)| \leq 7$, then $d(x)=d(y)=5,|N(x) \cap N(y)|=3$, and $G[N(x)], G[N(y)] \simeq K_{5}$.

The following theorem is the main result of this paper.

Theorem 6. If $G$ is a graph with treewidth at most 6 , then $\tau(G) \leq 2 \nu(G)$.

Proof. Suppose, for a contradiction, that the statement does not hold, and let $G$ be a minimal counterexample. It is not hard to check that $|V(G)| \geq 8$. We claim that $G$ is irreducible. Indeed, suppose that there is a set $V_{0} \subseteq V(G)$ that is reducible using $X$ and $Y$, and let $G^{\prime}=(G-X)-V_{0}$. Since $G^{\prime} \subseteq G$, we have $t w\left(G^{\prime}\right) \leq 6$, and by the minimality of $G$, we have $\tau\left(G^{\prime}\right) \leq 2 \nu\left(G^{\prime}\right)$. Thus, by Lemma $4, \tau(G) \leq 2 \nu(G)$, a contradiction. It is not hard to check that $G$ is also robust, and hence has minimum degree at least 5 .

By Proposition $1, G$ has a rooted tree decomposition $(T, \mathcal{V}, r)$. Since $|V(G)| \geq 8$, we have $|V(T)|>1$, and hence there is a node $t \in V(T)$ with $h(t)=1$. Suppose that $S(t)=\left\{t^{\prime}\right\}$, and $V_{t}=\left\{v_{t}, v_{1}, v_{2}, v_{3}, v_{4}, v_{5}, v_{6}\right\}$, where $v_{t}$ is the representative of $t$ if $t \neq r$. Since $(T, \mathcal{V}, r)$ is a rooted tree decomposition of $G$, we may assume that $V_{t^{\prime}}=\left\{v_{t}, v_{1}, v_{2}, v_{3}, v_{4}, v_{5}, x\right\}$, with $x \neq v_{6}$. By Proposition $2, d(x) \leq 6$, which implies, that $d(x) \in\{5,6\}$. Also, by Proposition 2 applied to $G-x$ and $t$, we have that $N_{G-x}\left(v_{t}\right) \subseteq V_{t}$. Thus $d\left(v_{t}\right)=\left|N\left(v_{t}\right)\right| \leq\left|N_{G-x}\left(v_{t}\right)\right|+1 \leq\left|V_{t}\right|=7$.

Suppose that $d\left(v_{t}\right)=7$. Now $N[x] \nsubseteq N\left[v_{t}\right]$ since $d(x) \in\{5,6\}$, either by Lemma 4(c) or by Lemma 4(d). But, since $d\left(v_{t}\right)=7$, note that $v_{t}$ is adjacent to $x$ and to every vertex in $V_{t} \backslash\left\{v_{t}\right\}$, hence $N[x] \subseteq N\left[v_{t}\right]$, a contradiction. So we may assume that $d\left(v_{t}\right) \leq 6$, and hence $v_{t} x \notin E(G)$ by Lemma $4(\mathrm{~b})$. Since $d\left(v_{t}\right) \geq 5$, there are at least four neighbors of $v_{t}$ in $\left\{v_{1}, v_{2}, v_{3}, v_{4}, v_{5}\right\}$, say $v_{1}, v_{2}, v_{4}, v_{5}$. Now, since $v_{t} x \notin E(G)$, we have $d(x)=5$. Thus, by Lemma 4(a), we have that $|E(G[N(x)])| \in\{9,10\}$. If $|E(G[N(x)])|=10$, then put

$X=\left(\left\{v_{t} v_{6}\right\} \cap E(G)\right) \cup E\left(G\left[\left\{v_{1}, v_{2}, v_{3}, v_{4}, v_{5}\right\}\right]\right)$ and $Y=\left\{v_{1} v_{2} v_{3}, v_{3} v_{4} v_{5}, v_{1} v_{4} x, v_{2} v_{5} x, v_{1} v_{5} v_{t}, v_{2} v_{4} v_{t}\right\}$.

Note that $|X| \leq 11 \leq 12=2|Y|$. It is not hard to check that $V_{0}=\left\{v_{t}, x\right\}$ is reducible using $X$ and $Y$, a contradiction. So we may assume that $|E(G[N(x)])|=9$. Note that the missing edge $e \notin E(G[N(v)])$ must be incident to a neighbor, say $v_{5}$, of $v_{t}$. Thus we may assume that $e \in\left\{v_{3} v_{5}, v_{4} v_{5}\right\}$. Put

$X=\left(\left\{v_{t} v_{6}\right\} \cap E(G)\right) \cup E\left(G\left[\left\{v_{1}, v_{2}, v_{3}, v_{4}, v_{5}\right\}\right]\right)$ and $Y=\left\{v_{1} v_{2} v_{3}, v_{1} v_{5} x, v_{3} v_{4} x, v_{1} v_{4} v_{t}, v_{2} v_{5} v_{t}\right\}$.

Note that $|X| \leq 10=2|Y|$. It is not hard to check that $V_{0}=\left\{v_{t}, x\right\}$ is reducible using $X$ and $Y$, a contradiction.

Therefore we may assume that $|S(t)|>1$. First, suppose that $|S(t)| \geq 3$, and let $t_{1}, t_{2}, t_{3} \in S(t)$, and vertices $x, y, z$ be the representatives of $t_{1}, t_{2}, t_{3}$, respectively. 
Note that, by Proposition 2, xy, xz,yz $\notin E(G)$ and $|N(x) \cup N(y)|,|N(x) \cup N(z)|$, and $|N(y) \cup N(z)| \leq\left|V_{t}\right|=7$. Thus, by Lemma $5, \quad d(x)=d(y)=d(z)=5$, $|N(x) \cap N(y)|=|N(x) \cap N(z)|=|N(y) \cap N(z)|=3$, and $G[N(x)], G[N(y)], G[N(z)] \simeq$ $K_{5}$. Assume without loss of generality that $N(x)=\left\{v_{1}, v_{2}, v_{3}, v_{4}, v_{5}\right\}$. Since $\mid N(x) \cap$ $N(y) \mid=3$, we may assume, without loss of generality, that $N(y)=\left\{v_{3}, v_{4}, v_{5}, v_{6}, v_{7}\right\}$. It is not hard to check that, since $|N(x) \cap N(z)|=|N(y) \cap N(z)|=3$ and $|N(z)|=5$, then $N(z)$ contains exactly one vertex in $N(x) \cap N(y)$. So we may assume, without loss of generality, that $N(z)=\left\{v_{1}, v_{2}, v_{4}, v_{6}, v_{7}\right\}$. Let $H=G\left[\left\{v_{1}, v_{2}, v_{3}, v_{4}, v_{5}, v_{6}, v_{7}\right\}\right]$ and note that every pair of vertices of $H$ is contained in at least one $N(v)$ for $v \in\{x, y, z\}$. Thus, since $G[N(v)]$ is isomorphic to $K_{5}$ for $v \in\{x, y, z\}, H$ is isomorphic to $K_{7}$. Let $X=E(H)$ and note that $|X|=21$. Put

$$
\begin{aligned}
& Y_{1}=\left\{x v_{1} v_{3}, x v_{2} v_{5}, y v_{3} v_{4}, y v_{5} v_{6}, z v_{1} v_{6}, z v_{2} v_{4}\right\} \\
& Y_{2}=\left\{v_{1} v_{2} v_{7}, v_{2} v_{3} v_{6}, v_{4} v_{6} v_{7}, v_{3} v_{5} v_{7}, v_{1} v_{4} v_{5}\right\}
\end{aligned}
$$

and note that if $Y=Y_{1} \cup Y_{2}$, then $|Y|=11$ and $|X| \leq 2|Y|$. Hence $V_{0}=\{x, y, z\}$ is reducible using $X$ and $Y$, a contradiction. We conclude that $|S(t)| \leq 2$.

If $t \neq r$, we let $w$ be the representative of $t$, otherwise we let $w$ be an arbitrary vertex of $V_{t}$. Let $t_{1}, t_{2} \in S(t)$ and let $x, y$ be the representatives of $t_{1}, t_{2}$, respectively. Again, by Lemma 5, we have $d(x)=d(y)=5,|N(x) \cap N(y)|=3$, and $G[N(x)], G[N(y)] \simeq$ $K_{5}$. Note that $t$ is a leaf of $\left(T^{\prime}, \mathcal{V}^{\prime}, r\right)$, where $T^{\prime}=T-t_{1}-t_{2}$ and $\mathcal{V}^{\prime}=\mathcal{V} \backslash\left\{V_{t_{1}}, V_{t_{2}}\right\}$, hence $d_{G-x-y}(w) \leq 6$. Thus, we have $d_{G}(w) \leq 8$. Note that $w \in N(x) \cup N(y)$ and assume, without loss of generality, that $w \in N(x)$. Since $G[N(x)]$ is a complete graph, we have $N[x] \subseteq N[w]$, a contradiction to Lemma 4(d). This concludes the proof.

\section{Concluding remarks}

This work has benefited greatly from [Puleo 2015]. Nevertheless, there were still gaps that we were able to explore. As we can see, the tree decomposition, specially under bounded treewidth, provides a suitable structure for problems of this nature. We believe that these techniques may be further improved by studying the behaviour of nodes from the tree decomposition with different heights.

\section{References}

Bodlaender, H. (1998). A partial $k$-arboretum of graphs with bounded treewidth. Theoretical Computer Science, 209(1):1-45.

Gross, J. (2014). Embeddings of graphs of fixed treewidth and bounded degree. ARS Mathematica Contemporanea, 7:379-403.

Haxell, P. E. and Kohayakawa, Y. (1998). Packing and covering triangles in tripartite graphs. Graphs and Combinatorics, 14(1):1-10.

Puleo, G. (2015). Tuza's conjecture for graphs with maximum average degree less than 7. European Journal of Combinatorics, 49:134-152.

Tuza, Z. (1981). Conjecture in: finite and infinite sets. In Proc. Colloq. Math. Soc. J. Bolyai (Eger, Hungary, 1981), volume 37, page 888.

Tuza, Z. (1990). A conjecture on triangles of graphs. Graphs and Combinatorics, $6(4): 373-380$. 